\title{
Left ventricular-arterial coupling as a predictor of stroke volume response to norepinephrine in septic shock - a prospective cohort study
}

Xiaoyang Zhou ${ }^{1,2}$, Jianneng Pan ${ }^{1,2}$, Yang Wang ${ }^{1,2}$, Hua Wang ${ }^{1,2}$, Zhaojun $\mathrm{Xu}^{1,2^{*}}$ and Weibo Zhuo ${ }^{3^{*}}$

\begin{abstract}
Background: Left ventricular-arterial coupling (VAC), defined as the ratio of arterial elastance (Ea) to left ventricular end-systolic elastance (Ees), is a key determinant of cardiovascular performance. This study aims to evaluate whether left VAC can predict stroke volume (SV) response to norepinephrine (NE) in septic shock patients.

Methods: This was a prospective cohort study conducted in an intensive care unit of a tertiary teaching hospital in China. We recruited septic shock patients who had persistent hypotension despite fluid resuscitation and required NE to maintain mean arterial pressure (MAP) $>65 \mathrm{mmHg}$. Those patients in whom the target MAP was not reached after NE infusion were ineligible. Echocardiographic variables were measured before (baseline) and after NE infusion. SV responder was defined by $a \geq 15 \%$ increase in SV after NE infusion.

Results: Of 34 septic shock patients included, 19 (56\%) were SV responders. Before NE infusion, SV responders had a lower Ees $(1.13 \pm 0.24 \mathrm{mmHg} / \mathrm{mL}$ versus $1.50 \pm 0.46 \mathrm{mmHg} / \mathrm{mL}, P=0.005)$ and a higher Ea/Ees ratio $(1.47 \pm 0.40$ versus $1.02 \pm 0.30, P=0.001)$ than non-responders, and Ea in SV responders was comparable to that in non-responders $(1.62 \pm$ $0.36 \mathrm{mmHg} / \mathrm{mL}$ versus $1.43 \pm 0.28 \mathrm{mmHg} / \mathrm{mL}, P=0.092)$. NE significantly increased Ea and Ees in both groups. The Ea/ Ees ratio was normalized by NE administration in SV responders but unchanged in non-responders. The baseline Ea/Ees ratio was positively correlated with NE-induced SV increases $(r=0.688, P<0.001)$. Logistic regression analysis indicated that the baseline Ea/Ees ratio was a predictor of SV increases induced by NE (odd ratio $0.008,95 \%$ confidence interval (Cl): 0.000 to 0.293 ), with an area under the receiver operating characteristic curve of 0.816 ( $95 \%$ Cl: 0.646 to 0.927 ).
\end{abstract}

Conclusions: The left VAC has the ability to predict SV response to NE infusion in septic shock patients.

Trial registration: Chinese Clinical Trial Registry, ChiCTR1900024031, Registered 23 June 2019 - Retrospectively registered, http://www.chictr.org.cn/edit.aspx?pid=40359\&htm $=4$.

Keywords: Septic shock, Stroke volume, Norepinephrine, Cardiovascular, Ventricular-arterial coupling

\footnotetext{
*Correspondence: nbey_icu@163.com; fhzyyicu@yeah.net

'Department of Intensive Care Medicine, HwaMei Hospital, University of

Chinese Academy of Sciences, Ningbo, Zhejiang 315000, China

${ }^{3}$ Department of Intensive Care Medicine, Ningbo Fenghua District Hospital of

Traditional Chinese Medicine Medical Community, Ningbo, Zhejiang 315500,

China

Full list of author information is available at the end of the article
}

(C) The Author(s). 2021 Open Access This article is licensed under a Creative Commons Attribution 4.0 International License, which permits use, sharing, adaptation, distribution and reproduction in any medium or format, as long as you give appropriate credit to the original author(s) and the source, provide a link to the Creative Commons licence, and indicate if changes were made. The images or other third party material in this article are included in the article's Creative Commons licence, unless indicated otherwise in a credit line to the material. If material is not included in the article's Creative Commons licence and your intended use is not permitted by statutory regulation or exceeds the permitted use, you will need to obtain permission directly from the copyright holder. To view a copy of this licence, visit http://creativecommons.org/licenses/by/4.0/. The Creative Commons Public Domain Dedication waiver (http://creativecommons.org/publicdomain/zero/1.0/) applies to the data made available in this article, unless otherwise stated in a credit line to the data. 


\section{Background}

Currently, septic shock remains the leading cause of death in the intensive care unit (ICU) with a high mortality of around 38\% [1]. Fluid administration is a very important treatment for septic shock, but it is always accompanied by an increased risk of fluid overload and seems to be insufficient to restore the arterial pressure due to the depressed vasomotor tone. Thus, vasopressor is advocated to be applied early to achieve a minimum acceptable arterial pressure to guarantee organ perfusion [2-4].

Norepinephrine (NE) is recommended as the first choice of vasopressor in the management of septic shock [5]. As a potent $\alpha 1$-adrenergic agent with $\beta 1$-adrenergic properties, NE can increase the left ventricular afterload and myocardial oxygen consumption through restoring vasomotor tone and subsequently improving arterial pressure $[6,7]$. On the other hand, NE can improve cardiac contractility through stimulating $\beta 1$-adrenergic receptors and improving coronary perfusion by increasing diastolic arterial pressure (DAP) [6], and it can also increase the left ventricular preload by redistributing venous blood from unstressed to stressed blood volume [2, 8, 9]. Given the wide spectrum of impacts of NE on cardiovascular performance, the overall cardiovascular effects of NE are difficult to determine.

It has been well described that the mechanical efficiency of the cardiovascular system depends on the interactions between the heart and the arterial system [10-12], namely left ventricular-arterial coupling (VAC), which is measured by the ratio of arterial elastance (Ea) to left ventricular end-systolic elastance (Ees). In the physiological conditions, the cardiac function is matched well with the arterial system, and this interaction is modulated dynamically to provide an optimal SV and arterial pressure to perfuse the organ and tissue [10]. However, this well-matched interaction will be inevitably broken in some pathological cases, such as septic shock [13], finally causing circulatory failure and worse prognosis [13-15]. Among interventions for the treatment of circulatory failure, the optimal treatment should be those that improve the work efficacy of the cardiovascular system with the lowest energetic consumption, which refers to high mechanical efficiency. Therefore, it is of interest to explore the effect of NE on the interactions between the heart and the arterial system, since NE exhibited complex effects on cardiovascular performance. Moreover, a description of the cardiovascular effects of NE will facilitate a better understanding of the pathophysiologic changes of hemodynamics during NE infusion. We therefore conducted this study to describe the relationship between the left VAC and the cardiovascular response to NE in septic shock patients. We hypothesized that the left VAC can predict SV response to NE in septic shock, given the fact that the left VAC determines the stroke volume (SV), left ventricular ejection fraction (LVEF), and ejection pressure $[10,16]$, and it possesses independent diagnostic and prognostic value in multiple diseases [17].

\section{Materials and methods}

This was a prospective cohort study conducted between October 2018 and January 2020 in the 20-bed ICU of HwaMei Hospital, University of Chinese Academy of Sciences (Ningbo, China). This study was conducted in compliance with the Declaration of Helsinki and approved by the institutional ethics committee in our hospital (PJ-NBEY -KY-2019-014-01) and adhered to the Strengthening the Reporting of Observational Studies in Epidemiology (STROBE) guidelines. Written informed consent was obtained from the patients or their next of kin. This study was part of a study program that was registered in the Chinese Clinical Trial Registry (ChiCTR1900024031).

\section{Patients}

Adult patients (age $>18$ years) with septic shock, who had persistent hypotension despite fluid resuscitation and required $\mathrm{NE}$ to maintain mean arterial pressure (MAP) $>65 \mathrm{mmHg}$, were considered for enrollment after ICU admission. Septic shock was diagnosed according to the criteria of the third international consensus definitions for sepsis and septic shock [18]. The exclusion criteria included: 1) Refractory shock patients in whom the target MAP was not reached after NE infusion and needed to infuse other vasopressors or inotropic agents to maintain MAP; 2) Patients with atrial fibrillation; 3) Patients who were receiving vasoactive agents or cardiac function assist device (such as pacemaker) at the time of enrollment; 4) Patients who had poor echogenicity or could not tolerate the transthoracic echocardiography (TTE) examination.

\section{Study protocol}

Radial artery catheterization was performed in all patients after their ICU admission to measure the invasive arterial pressure. The initial resuscitation practice adhered to the recommendations of the Surviving Sepsis Campaign [5] and its update [4]. These practices included fluid resuscitation, appropriate antibiotic therapy, source control, vasoactive medications, and organ support. Fluid responsiveness was evaluated using dynamic echocardiographic indices (e.g. the respiratory variation in inferior vena cava diameter, the passive leg raisinginduced changes in SV) before NE infusion start. Whether start NE infusion was decided by the physician in charge based on the MAP, fluid non-responsiveness, and fluid volume administered in each patient (at least $30 \mathrm{~mL} / \mathrm{kg}$ of crystalloid fluid within the first $3 \mathrm{~h}$ ). NE 
dose was adjusted to reach the target MAP (more than $65 \mathrm{mmHg}$ ) and maintain MAP stabilization. MAP stabilization was defined as a variation of MAP $<10 \%$ with NE infusion during a period of at least $15 \mathrm{~min}$ [19]. Other vasoactive drugs or inotropic agents were not considered before the end of the study period. Additional sedative and analgesic drugs were used to facilitate invasive mechanical ventilation (IMV) in patients treated with IMV. Modifications of ventilator setting or dose of sedative and analgesic drugs and fluid challenges were not allowed during the study period.

\section{Data collection}

We recorded the demographic information, source of infection, causative pathogen in culture, and concomitant disease for all patients at ICU admission. The blood gas, acute physiology and chronic health evaluation (APAC $\mathrm{HE)}$ II score, and sequential organ failure assessment (SOFA) score at the time of enrolment were also collected for each patient. Central venous pressure (CVP) was measured before and after $\mathrm{NE}$ infusion for all subjects. The ratio of arterial oxygen partial pressure $\left(\mathrm{PaO}_{2}\right)$ to fractional inspired oxygen $\left(\mathrm{FiO}_{2}\right)$, ventilator parameters, type of sedative and analgesic drug, and length of IMV were collected for patients treated with IMV. Finally, we recorded and analyzed the dose of NE administered, urine output, the time elapsed from NE infusion start to MAP stabilization, duration of ICU stay, and cumulative fluid volume (before NE infusion, during the study period, and within the first $24 \mathrm{~h}$ after septic shock diagnosis). All patients were followed up to hospital discharge.

\section{Transthoracic echocardiography}

TTE examination was performed for all patients by an independent ICU physician using a Philips CX50 ultrasound system (Philips Medical System, Suresnes, France). This trained operator had an operating experience in TTE for more than 3 years and was blinded to our study protocol. The left lateral decubitus position was preferred to obtain a good cardiac ultrasound image. All patients were connected to an electrocardiogram.

In the apical four-chamber view, left ventricular enddiastolic volume (LVEDV) and left ventricular endsystolic volume (LVESV) were measured using Simpson's method, then LVEF was calculated. Continuous Doppler transaortic flow was obtained from the apical five-chamber view to measure the aortic velocity-time integral (VTI), pre-ejection time $\left(\mathrm{T}_{\text {pre-e }}\right)$, and total systolic time $\left(\mathrm{T}_{\text {tot-s }}\right)$. The diameter of the left ventricular outflow tract (LVOT) was measured in the parasternal long-axis view, and the area of LVOT was then calculated. Simultaneously, heart rate (HR), systolic arterial pressure (SAP), and DAP, as well as MAP, were also measured at the time of TTE examination. Finally, SV was calculated using the formula: $\mathrm{SV}=\mathrm{VTI} \times \mathrm{LVOT}$ area, and cardiac output $(\mathrm{CO})$ was calculated as $\mathrm{SV} \times$ HR. Ea was estimated as $(0.9 \times \mathrm{SAP}) / \mathrm{SV}$ [20], and Ees was calculated using the single-beat method proposed by Chen et al. [21]. According to the previous publications $[22,23], \mathrm{Ea} /$ Ees $>1.36$ was considered as left ventriculararterial uncoupling.

All measurements were performed at two time points: starting NE infusion (before NE infusion, baseline) and immediately after MAP stabilization (after NE infusion), regardless of the respiratory cycle. The representative value for each variable was estimated as the average value of three consecutive measurements. The NE-induced SV increase was employed to distinguish the SV responder (NE-induced SV increases $\geq 15 \%$ ) from non-responder (NE-induced increases $<15 \%$ ), where the NE-induced SV increase was calculated as (SV after NE infusion - SV before NE infusion)/ SV before NE infusion $\times 100 \%$.

\section{Statistical analysis}

The distribution of continuous variables was tested for normality using the Kolmogorov-Smirnov test. Normally distributed variables were expressed as mean \pm standard deviation (SD), and variables with skewed distribution were presented as median and interquartile range (IQR). Categorical variables were expressed as frequency and percentages. Comparisons between SV responders and non-responders were assessed using the Student $\mathrm{t}$ test, Mann-Whitney $\mathrm{U}$ test, or Fisher exact test, as appropriate. Comparisons between the two time points within a group were assessed using the Student paired $t$ test. The log-rank test was used to compare hospital mortality between the two groups. Pearson correlation coefficient was calculated to test the relationship between the baseline Ea/Ees ratio and other cardiovascular variables (including HR, SAP, CVP, LVEDV, LVEF, $\mathrm{SV}$, and NE-induced SV increases) and to investigate whether NE-induced changes in Ea depend more on changes in SAP or SV. Univariate logistic regression analyses were used to screen the potential predictors of SV increase induced by NE. Given the small sample size, multivariate analysis was not performed. Receiver operating characteristic (ROC) curve was constructed for the $\mathrm{Ea} /$ Ees ratio, SV, SAP, and LVEDV at baseline to discriminate the SV responder from SV non-responder, and the optimal cutoff value was determined by the maximum of Youden index.

A sample size of 34 subjects was calculated to have a power of at least $90 \%$ to prove the hypothesis that the baseline $\mathrm{Ea} /$ Ees ratio could predict an increase in SV of $\geq 15 \%$ in response to NE with an area under the ROC curve (AUC) of $0.8, \alpha$ of 0.05 . The coefficient of variation $(\mathrm{CV})$ and least significant change (LSC) were 
calculated to assess the intra-observer reproducibility for these directly measured ultrasound variables, including LVEDV, LVESV, VTI, $\mathrm{T}_{\text {pre-e }}$ and $\mathrm{T}_{\text {tot-s }}$, in 10 randomly selected patients. Two-sided $P$ value $<0.05$ was considered as statistical significance. Data analyses were performed using the statistical software SPSS 17.0 (IBM, New York, USA).

\section{Results}

A total of 38 septic shock patients were initially consecutively screened for enrollment. After excluding 4 ineligible patients, we included 34 subjects, of which 19 were SV responders and 15 were SV non-responders (Fig. 1). The demographic characteristics of the included patients are summarized in details in Table 1 . The baseline characteristics of responders were comparable to that of non-responders. Most of the included patients (71\%) received IMV during the study period, and the duration of IMV was similar between groups. SV non-responders probably received more fluid during the first $24 \mathrm{~h}$ after the onset of septic shock than responders $(P=0.061)$. However, the cumulative fluid volumes before NE infusion and during the study period were similar between groups. The duration of ICU stay and in-hospital mortality did not differ between groups. In the whole studied population, the average value of Ea/Ees ratio before NE infusion was $1.27 \pm 0.42$, and 10 patients $(29 \%)$ had an uncoupled ventricular-arterial interaction with an $\mathrm{Ea} / \mathrm{Ees}$ ratio of $>1.36$.

\section{Intra-observer reproducibility}

As shown in Table 2, the intra-observer reproducibility for the directly measured ultrasound variables was acceptable.

\section{Cardiovascular response to norepinephrine infusion}

Before NE infusion, SV responders had a lower VTI, lower LVEF, and higher LVESV than non-responders. The HR, SAP, DAP, MAP, CVP, SV, LVEDV, cardiac index, $\mathrm{T}_{\text {pre-e, }}$ and $\mathrm{T}_{\text {tot-s }}$ in responders were comparable to that in non-responders (all $P>0.05$ ). Although Ea did not differ between groups, Ees in responders was significantly lower than that in non-responders $(P=0.005)$, thus resulted in a higher $\mathrm{Ea} /$ Ees ratio in responders than non-responders $(P=0.001)$ (Table 3 , Fig. 2$)$.

In both groups, NE significantly increased the SV. The NE-induced SV increases in responders were greater than that in non-responders $(21.1 \pm 5.4 \%$ versus $5.8 \pm 5.5 \%, P<0.001)$. Both Ea and Ees were increased by NE in both groups, and the increases in Ea were lower in responders $(0.17 \pm 0.22 \mathrm{mmHg} / \mathrm{mL}$ versus $0.39 \pm 0.22 \mathrm{mmHg} / \mathrm{mL}, \quad P=0.008)$. However, the NE-induced increases of Ees in responders did not differ from that of non-responders $(0.37 \pm 0.26$ $\mathrm{mmHg} / \mathrm{mL}$ versus $0.32 \pm 0.21 \mathrm{mmHg} / \mathrm{mL}, P=0.577$ ), thus $\mathrm{Ea} /$ Ees was normalized by $\mathrm{NE}$ in responders, while unchanged in non-responders (Table 3). The individual data on the Ea/Ees ratio for each patient is shown in Fig. 3.

NE also increased the SAP, DAP, and MAP in both groups. Besides, the HR was reduced by NE infusion in both groups. Accordingly, NE induced a significant increase in the $\mathrm{T}_{\text {tot-s}}$, but the $\mathrm{T}_{\text {pre-e }}$ was unchanged. Additionally, the administration of NE was associated with an increase in the LVEDV and VTI in both groups, but not the CVP. However, NE infusion resulted in a decrease of LVESV in responders, but not in nonresponders. Thus, the LVEF and cardiac index were improved by $\mathrm{NE}$ in responders, yet not changed in nonresponders (Table 3 ).

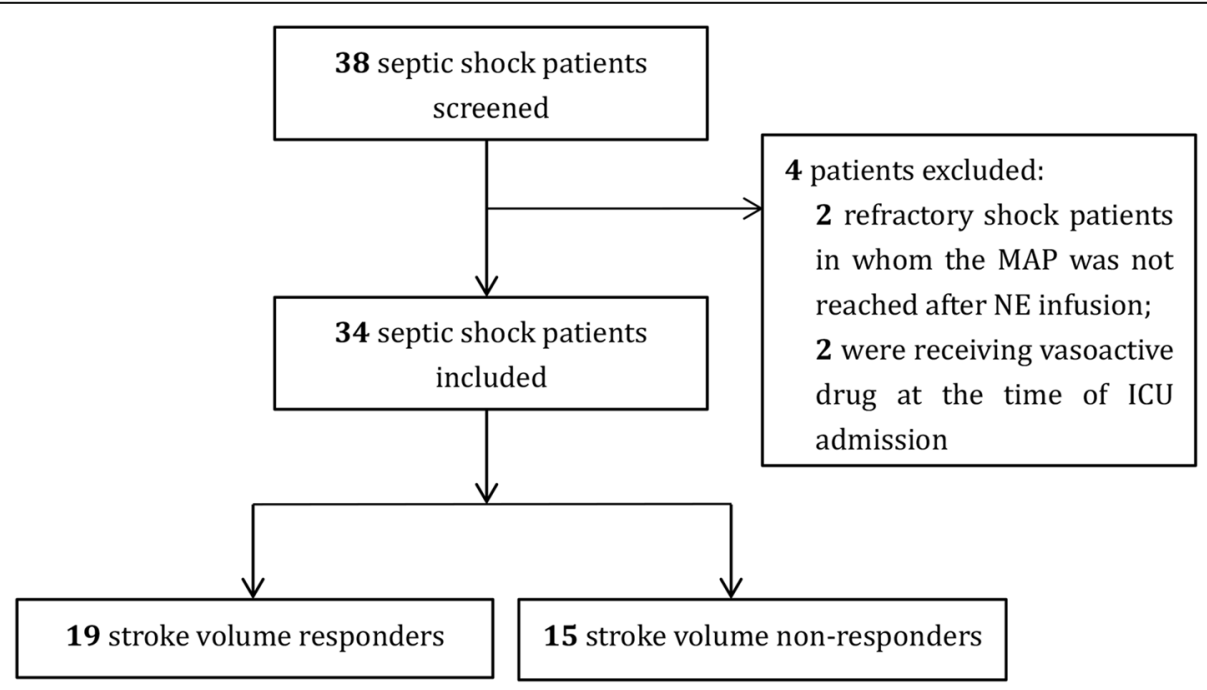

Fig. 1 Flow chart of this study. NE norepinephrine; ICU intensive care unit 
Table 1 Clinical characteristics and demographic data of the study participants

\begin{tabular}{|c|c|c|c|c|}
\hline Variables & $\begin{array}{l}\text { All patients } \\
(n=34)\end{array}$ & $\begin{array}{l}\text { SV responders } \\
(n=19)\end{array}$ & $\begin{array}{l}\text { SV non-responders } \\
(n=15)\end{array}$ & $P$ value $^{a}$ \\
\hline Age (years, mean $\pm S D$ ) & $70 \pm 12$ & $69 \pm 13$ & $73 \pm 11$ & 0.368 \\
\hline Gender [Male, n (\%)] & $24(71 \%)$ & $14(74 \%)$ & $10(67 \%)$ & 0.718 \\
\hline Body mass index $\left(\mathrm{kg} / \mathrm{m}^{2}\right.$, mean $\left.\pm \mathrm{SD}\right)$ & $22.5 \pm 3.1$ & $22.6 \pm 3.1$ & $22.5 \pm 3.3$ & 0.932 \\
\hline Body surface area $\left(m^{2}\right.$, mean $\left.\pm S D\right)$ & $1.65 \pm 0.16$ & $1.68 \pm 0.17$ & $1.62 \pm 0.16$ & 0.300 \\
\hline APACHE II score (mean \pm SD) & $20 \pm 6$ & $21 \pm 5$ & $20 \pm 6$ & 0.644 \\
\hline SOFA score $($ mean \pm SD) & $9 \pm 3$ & $9 \pm 3$ & $8 \pm 2$ & 0.554 \\
\hline \multicolumn{5}{|l|}{ Source of infection, n (\%) } \\
\hline Lung & $18(53 \%)$ & $11(58 \%)$ & $7(47 \%)$ & 0.730 \\
\hline Urinary tract & $7(21 \%)$ & $3(16 \%)$ & $4(27 \%)$ & 0.672 \\
\hline Abdomen & $7(21 \%)$ & $5(26 \%)$ & $2(13 \%)$ & 0.426 \\
\hline Bloodstream & $7(21 \%)$ & $3(16 \%)$ & $4(27 \%)$ & 0.672 \\
\hline Others & $4(12 \%)$ & $2(11 \%)$ & $2(13 \%)$ & 1.000 \\
\hline \multicolumn{5}{|l|}{ Co-morbidities, n (\%) } \\
\hline Hypertension & $16(47 \%)$ & $9(47 \%)$ & $7(47 \%)$ & 1.000 \\
\hline Diabetes & $10(29 \%)$ & $7(37 \%)$ & $3(20 \%)$ & 0.451 \\
\hline Chronic obstructive pulmonary disease & $3(9 \%)$ & $2(11 \%)$ & $1(7 \%)$ & 1.000 \\
\hline Coronary heart disease & $2(6 \%)$ & $2(11 \%)$ & $0(0 \%)$ & 0.492 \\
\hline Chronic kidney disease & $2(6 \%)$ & $1(5 \%)$ & $1(7 \%)$ & 1.000 \\
\hline \multicolumn{5}{|l|}{ Pathogen type in culture, $\mathrm{n}(\%)$} \\
\hline Gram-negative & $15(44 \%)$ & $9(47 \%)$ & $6(40 \%)$ & \multirow[t]{5}{*}{0.624} \\
\hline Gram-positive & $1(3 \%)$ & $1(5 \%)$ & $0(0 \%)$ & \\
\hline Mixed & $2(6 \%)$ & $1(5 \%)$ & $1(7 \%)$ & \\
\hline Fungus & $2(6 \%)$ & $0(0 \%)$ & $2(13 \%)$ & \\
\hline No pathogen & $14(41 \%)$ & $8(42 \%)$ & $6(40 \%)$ & \\
\hline Patients receiving IMV, n (\%) & $24(71 \%)$ & $15(79 \%)$ & $9(60 \%)$ & 0.276 \\
\hline $\mathrm{PaO}_{2} / \mathrm{FiO}_{2}($ mean $\pm \mathrm{SD})$ & $262 \pm 135$ & $248 \pm 134$ & $285 \pm 142$ & 0.518 \\
\hline PEEP (cm H2O, mean \pm SD) & $6 \pm 1$ & $5 \pm 1$ & $6 \pm 2$ & 0.479 \\
\hline Tidal volume (mL/kg of predicted body weight, mean \pm SD) & $7.1 \pm 1.3$ & $6.8 \pm 1.2$ & $7.6 \pm 1.3$ & 0.129 \\
\hline Fentanyl, n (\%) & $15(44 \%)$ & $10(53 \%)$ & $5(33 \%)$ & 0.314 \\
\hline Midazolam, n (\%) & $15(44 \%)$ & $10(53 \%)$ & $5(33 \%)$ & 0.314 \\
\hline Propofol, n (\%) & $18(53 \%)$ & $11(58 \%)$ & $7(47 \%)$ & 0.730 \\
\hline Duration of IMV [days, median (IQR)] & $6(3-12)$ & $6(3-13)$ & $5(3-16)$ & 0.719 \\
\hline Serum lactate level (mmol/L, mean $\pm \mathrm{SD})$ & $3.5 \pm 2.7$ & $2.9 \pm 1.4$ & $4.2 \pm 3.7$ & 0.194 \\
\hline Patients with left ventricular-arterial uncoupling before NE infusion, n (\%) & $10(29 \%)$ & $8(42 \%)$ & $2(13 \%)$ & 0.128 \\
\hline Time from NE infusion start to MAP stabilization [min, median (IQR)] & $95(39-158)$ & $86(37-180)$ & $100(55-135)$ & 0.862 \\
\hline Cumulative fluid volume before NE infusion ( $\mathrm{mL}$, mean \pm SD) & $1638 \pm 569$ & $1542 \pm 523$ & $1758 \pm 620$ & 0.278 \\
\hline Cumulative fluid volume during the study period [mL, median (IQR)] & $193(90-309)$ & $180(60-350)$ & $210(120-305)$ & 0.490 \\
\hline Cumulative fluid volume within the first $24 \mathrm{~h}(\mathrm{~mL}$, mean \pm SD) & $3744 \pm 1251$ & $3389 \pm 1181$ & $4194 \pm 1228$ & 0.061 \\
\hline NE dose $(\mu \mathrm{g} / \mathrm{kg} / \mathrm{min}$, median (IQR)) & $0.254(0.131-0.556)$ & $0.22(0.09-0.556)$ & $0.44(0.182-0.556)$ & 0.167 \\
\hline Urine output (mL/kg/h, mean \pm SD) & $1.32 \pm 0.73$ & $1.13 \pm 0.41$ & $1.55 \pm 0.97$ & 0.133 \\
\hline Duration of ICU stay [days, median (IQR)] & $7(5-16)$ & $7(6-16)$ & $12(4-17)$ & 0.958 \\
\hline In-hospital mortality, n (\%) & $9(26 \%)$ & $6(32 \%)$ & $3(20 \%)$ & 0.485 \\
\hline
\end{tabular}

${ }^{a} P$ value for comparisons of SV responders and SV non-responders

SV stroke volume; APACHE acute physiology and chronic health evaluation; SOFA sequential organ failure assessment; IMV invasive mechanical ventilation; $\mathrm{PaO}_{2}$ arterial oxygen partial pressure; $\mathrm{FiO}_{2}$ fractional inspired oxygen; PEEP positive end-expiratory pressure; NE norepinephrine; MAP mean arterial pressure; ICU intensive care unit; SD standard deviation; IQR interquartile range 


\section{Pearson correlation and logistic regression analysis}

At baseline, the Ea/Ees ratio was positively correlated with the NE-induced SV increases $(r=0.688, P<0.001)$, and was negatively correlated with the LVEF $(r=-$ $0.809, P<0.001)$ and SV $(r=-0.560, P=0.001)$. The $\mathrm{Ea} /$ Ees ratio had no correlations with the HR, SAP, CVP, or LVEDV (all $P>0.05$ ). In addition, the NE-induced changes in Ea seem to be more related to the changes in SAP $(r=0.802, P<0.01)$ than that in SV $(r=-0.394$, $P=0.021)$.

In the univariate logistic regression analysis, the baseline $\mathrm{Ea} /$ Ees ratio was identified as a potential predictor of SV response to NE $(P=0.009)$ (Table 4).

\section{Receiver operating characteristic curve}

The ROC curves analyses suggested that the baseline $\mathrm{Ea}$ /Ees ratio could predict an increase $\geq 15 \%$ in SV after NE infusion, with an AUC of 0.816 (95\% CI: 0.646 to $0.927, P<0.001$ ) (Fig. 4). The optimal cutoff value was 1.11, with a sensitivity of $89.5 \%$ (95\% CI: 66.9 to $98.7 \%$ ), a specificity of $60.0 \%$ (95\% CI: 32.3 to $83.7 \%$ ), a positive likelihood ratio of 2.24 (95\% CI: 1.2 to 4.2$)$, and a negative likelihood ratio of 0.18 (95\% CI: 0.04 to 0.7 ). However, the baseline SV, SAP, and LVEDV had no ability to predict the SV response to NE, with an AUC of 0.626 (95\% CI: 0.444 to $0.786, P=0.218$ ), 0.626 (95\% CI: 0.444 to $0.786, P=0.192$ ), and 0.593 (95\% CI: 0.412 to 0.758 , $P=0.353)$, respectively.

\section{Discussion}

This study was conducted to evaluate the predictive value of left VAC for the SV response to NE in septic shock patients. The results suggested that SV responders had an altered baseline left VAC, which was significantly greater than that in SV non-responders, and the baseline left VAC was positively correlated with the NE-induced SV increases. This study found that the baseline left VAC had the ability to predict SV response to NE infusion in septic shock patients, and the NE-induced SV increase was due to the normalization of left VAC, which was mainly attributed to the improvement of Ees rather than Ea.

Additionally, the current study suggested that both VTI and Ees were improved after NE infusion, indicating an improvement in cardiac contractility, which was consistent with the findings in a previous study [24]. However, the LVEF was not simultaneously improved in the non- responder group. This result is not surprising, because LVEF is not a reliable index of cardiac contractility, and it also depends on the ventricular afterload [23]. Several studies $[25,26]$ had demonstrated that fluid responsiveness was a factor that influenced the effects of various interventions on the left VAC. These studies $[25,26]$ found an increase in SV and a decrease in Ea, resulting in an improved left VAC, after fluid loading in fluid responders. Thus, confirmation of fluid non-responsiveness before NE infusion start was an important process in our study. Moreover, we did not allow the fluid challenge during the study period. Finally, the cumulative volume of fluid infusion during NE infusion was small, and it was similar between responders and non-responders. However, we found a significant increase in LVEDV in both groups. It could not conclude that NE increased the ventricular preload, because the small changes in LVEDV were probably not clinically relevant. Thus, the small fluid volume administered during NE infusion should have, if have to be considered, a very limited impact on our results.

Given that the changes in Ea and Ees were largely different between SV responders and non-responders, we speculated that the SV responsiveness to NE might be determined by the comprehensive effects of NE on the left VAC. In our study, we found that SV nonresponders had a normal left VAC, Ea, and Ees at baseline. Administration of NE induced a similar improvement in both Ea and Ees, resulting in an unchanged left VAC, thus the potential increase in SV might be counterbalanced by the NE-induced increase in Ea which means a proportional increase in the end-systolic pressure (ESP) at a given SV. On the contrary, SV responders had an abnormal baseline left VAC (Ea/Ees ratio $>1.36$ ) that mainly resulted from impaired Ees. After NE administration, the left VAC was normalized, and it was mainly attributed to the improvement of Ees rather than Ea. The large improvement in Ees finally caused a significant increase in SV despite the small increase of Ea. These findings indicated that NE seemingly exerted a main inotropic effect in patients with abnormal left VAC, and exerted similar inotropic and vasoconstrictive effects in patients with normal left VAC. Furthermore, the comprehensive effect of NE on the interaction between cardiac and arterial performance was determined by the baseline left VAC. Our study suggested the ability of the baseline left VAC to predict the

Table 2 Intra-observer reproducibility for directly measured ultrasound variables

\begin{tabular}{llllll}
\hline Variables & LVEDV & LVESV & VTI & T $_{\text {pre-e }}$ & $\mathbf{T}_{\text {tot-s }}$ \\
\hline $\mathrm{CV}(\%, 95 \mathrm{Cl})$ & $3.6(2.8-4.5)$ & $4.7(3.7-5.7)$ & $1.9(1.3-2.5)$ & $6.1(3.9-8.4)$ & $2.3(1.7-2.9)$ \\
LSC (\%, 95 Cl) & $5.8(4.5-7.2)$ & $7.6(6.0-9.2)$ & $3.0(2.1-4.0)$ & $9.8(6.2-13.4)$ & $3.6(2.7-4.6)$ \\
\hline
\end{tabular}

CV coefficient of variation; LSC least significant change; LVEDV left ventricular end-diastolic volume; LDESV left ventricular end-systolic volume; VTI velocity-time integral; $T_{\text {pre-e }}$ pre-ejection time; $T_{\text {tot-s }}$ total systolic time; $C$ confidence interval 
Table 3 Cardiovascular responses to norepinephrine in stroke volume responders and non-responders

\begin{tabular}{|c|c|c|c|c|c|c|}
\hline \multirow[t]{2}{*}{ Variables } & \multicolumn{2}{|c|}{ SV responders $(n=19)$} & \multicolumn{2}{|c|}{ SV non-responders $(n=15)$} & \multirow{2}{*}{$\begin{array}{l}P \\
\text { value } \\
\mathrm{a}\end{array}$} & \multirow[t]{2}{*}{$P$ value } \\
\hline & Before NE & After NE & Before NE & After NE & & \\
\hline HR (beats/min) & $112 \pm 19$ & $104 \pm 20^{c}$ & $106 \pm 18$ & $96 \pm 21^{c}$ & 0.384 & 0.255 \\
\hline $\mathrm{SAP}(\mathrm{mmHg})$ & $84 \pm 6$ & $112 \pm 14^{d}$ & $81 \pm 6$ & $109 \pm 11^{d}$ & 0.247 & 0.456 \\
\hline $\mathrm{DAP}(\mathrm{mmHg})$ & $48 \pm 5$ & $64 \pm 9^{d}$ & $47 \pm 5$ & $56 \pm 5^{d}$ & 0.535 & 0.006 \\
\hline $\operatorname{MAP}(\mathrm{mmHg})$ & $60 \pm 4$ & $80 \pm 9^{d}$ & $59 \pm 4$ & $73 \pm 5^{d}$ & 0.269 & 0.027 \\
\hline CVP $(\mathrm{mmHg})$ & $9 \pm 4$ & $10 \pm 3$ & $8 \pm 5$ & $9 \pm 3$ & 0.498 & 0.329 \\
\hline VTI (cm) & $16.9 \pm 3.2$ & $20.3 \pm 3.3^{d}$ & $20.3 \pm 4.4$ & $21.4 \pm 4.1^{c}$ & 0.012 & 0.380 \\
\hline $\mathrm{SV}(\mathrm{mL})$ & $48 \pm 10$ & $58 \pm 11^{d}$ & $53 \pm 12$ & $56 \pm 11^{c}$ & 0.219 & 0.534 \\
\hline LVEDV (mL) & $100 \pm 12$ & $104 \pm 12^{d}$ & $95 \pm 13$ & $98 \pm 12^{c}$ & 0.261 & 0.145 \\
\hline LVESV (mL) & $52 \pm 7$ & $48 \pm 6^{d}$ & $43 \pm 10$ & $43 \pm 9$ & 0.004 & 0.061 \\
\hline LVEF (\%) & $47 \pm 6$ & $54 \pm 6^{d}$ & $54 \pm 8$ & $56 \pm 8$ & 0.006 & 0.255 \\
\hline Cardiac index $\left(\mathrm{L} / \mathrm{min} / \mathrm{m}^{2}\right)$ & $3.2 \pm 0.9$ & $3.6 \pm 1.0^{d}$ & $3.5 \pm 0.8$ & $3.3 \pm 0.8$ & 0.440 & 0.276 \\
\hline $\mathrm{T}_{\text {pre-e }}(\mathrm{ms})$ & $61 \pm 17$ & $59 \pm 14$ & $53 \pm 17$ & $56 \pm 14$ & 0.145 & 0.587 \\
\hline $\mathrm{T}_{\text {tot-s }}(\mathrm{ms})$ & $227 \pm 45$ & $246 \pm 44^{c}$ & $253 \pm 40$ & $280 \pm 41^{e}$ & 0.083 & 0.029 \\
\hline $\mathrm{Ea}(\mathrm{mmHg} / \mathrm{mL})$ & $1.62 \pm 0.36$ & $1.79 \pm 0.42^{c}$ & $1.43 \pm 0.28$ & $1.81 \pm 0.40^{d}$ & 0.092 & 0.877 \\
\hline Ees $(\mathrm{mmHg} / \mathrm{mL})$ & $1.13 \pm 0.24$ & $1.50 \pm 0.39^{d}$ & $1.50 \pm 0.46$ & $1.82 \pm 0.56^{d}$ & 0.005 & 0.057 \\
\hline Ea/Ees ratio & $1.47 \pm 0.40$ & $1.24 \pm 0.32^{d}$ & $1.02 \pm 0.30$ & $1.06 \pm 0.34$ & 0.001 & 0.145 \\
\hline
\end{tabular}

The data are presented as mean \pm standard deviation

${ }^{\text {a }} P$ value for comparisons of SV responders and non-responders before NE infusion; ${ }^{b} P$ value for comparisons of SV responders and non-responders after NE infusion; ${ }^{c} P<0.01,{ }^{d} P<0.001$, and ${ }^{\mathrm{e}} P<0.05$ for comparisons of before and after NE infusion within group

SV stroke volume; NE norepinephrine; HR heart rate; SAP systolic arterial pressure; DAP diastolic arterial pressure; MAP mean arterial pressure; CVP central venous pressure; VTI velocity-time integral; LVEDV left ventricular end-diastolic volume; LDESV left ventricular end-systolic volume; LVEF left ventricular ejection fraction;

$\mathrm{T}_{\text {pre-e }}$ pre-ejection time; $\mathrm{T}_{\text {tot-s }}$ total systolic time; Ea effective arterial elastance; Ees left ventricular effective end-systolic elastance

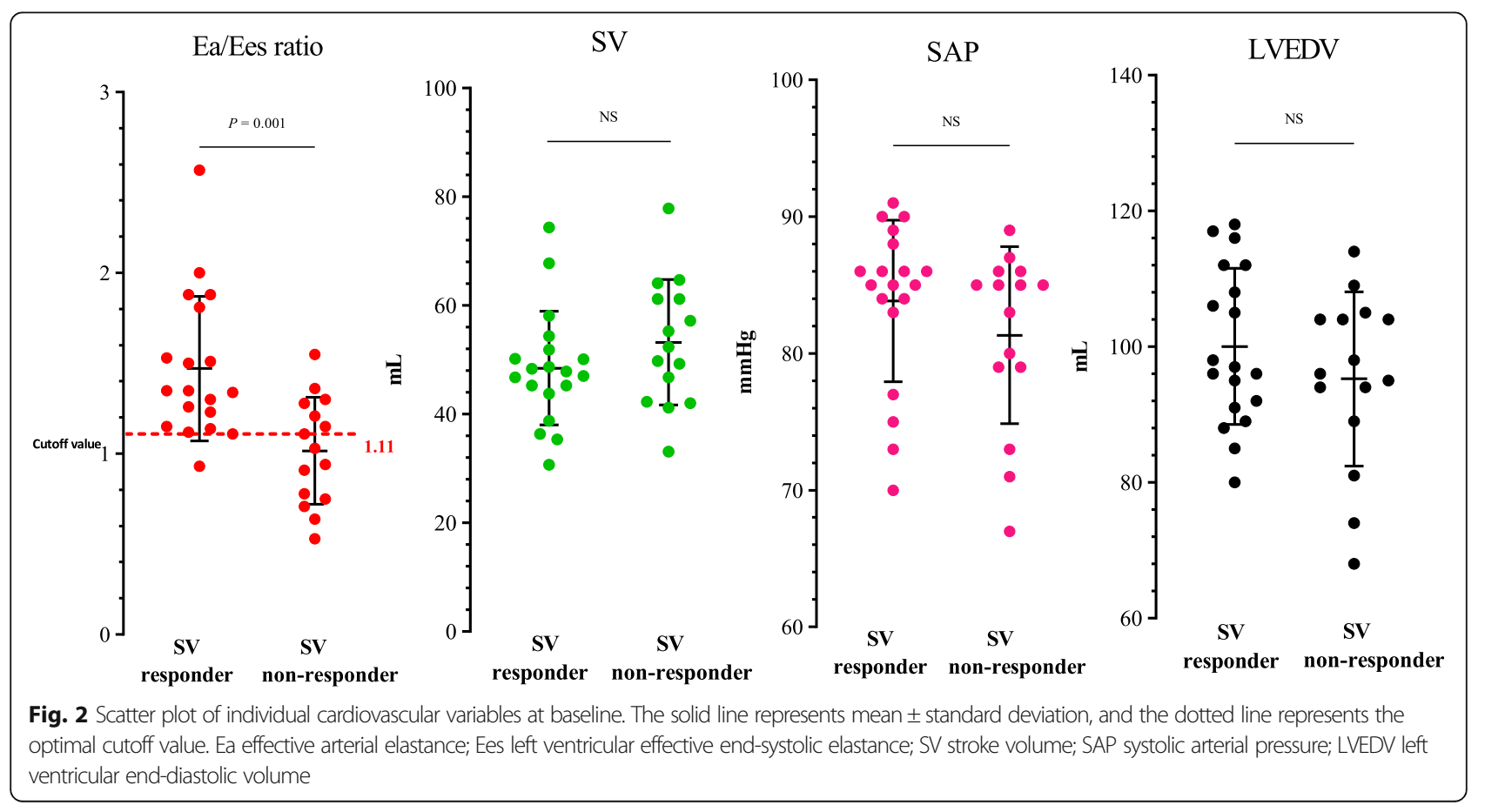




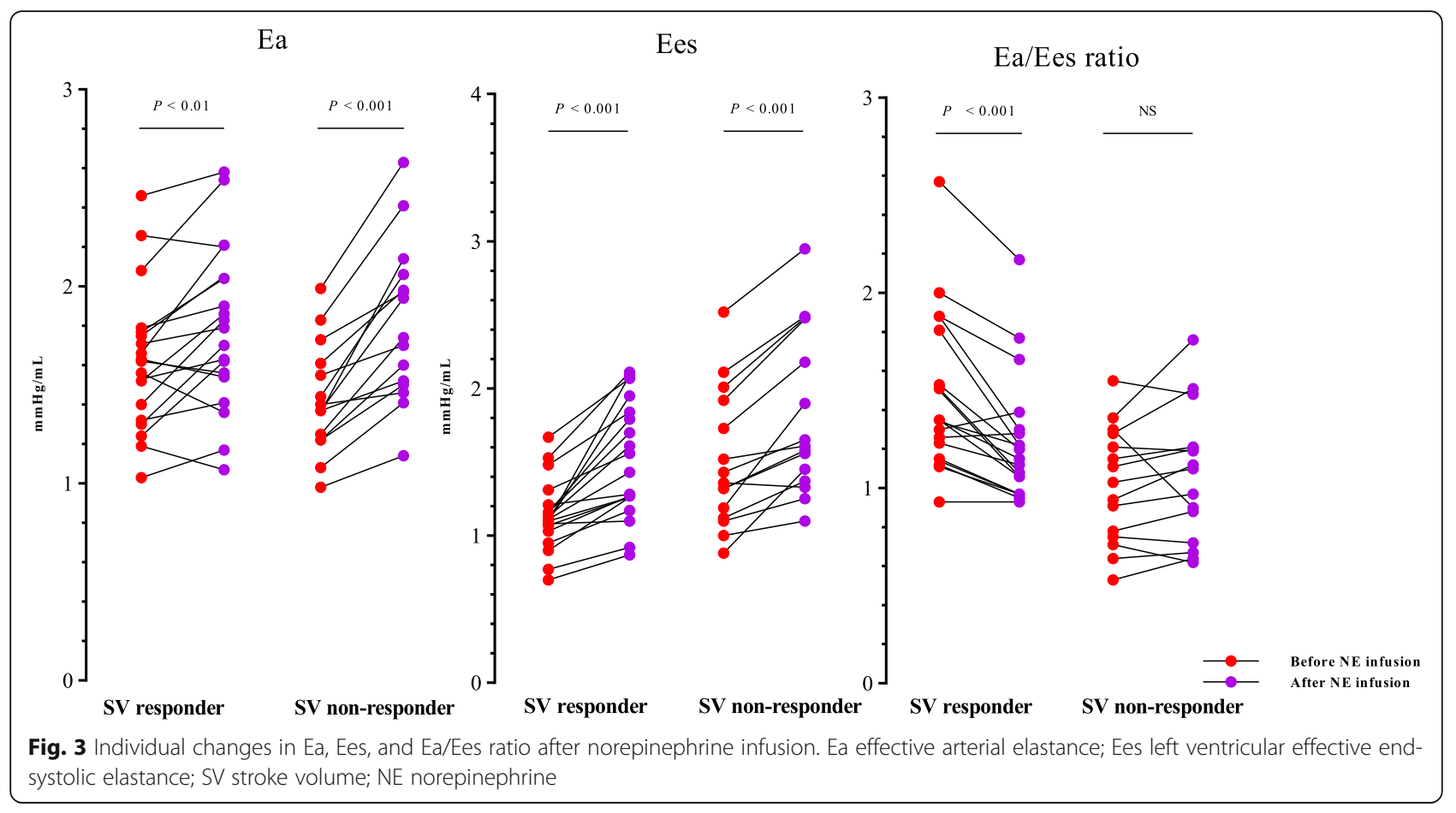

SV response to NE infusion, which was consistent with the result from the study by Guinot et al. [19]. Differently, the study by Guinot et al. [19] recruited postcardiac surgery patients who usually have low $\mathrm{CO}$ and high peripheral vascular resistance, which is different from the hemodynamic profile of septic shock that generalized vasodilation resulting in high $\mathrm{CO}$ with or without myocardial depression.
Over past decades, Ea has been widely recognized as a measure of ventricular afterload [20, 27]. According to the calculation formula, Ea is the change in ESP for a given change in SV, and it reflects all the extracardiac forces opposing to ventricular ejection [27]. Of note, a previous study [26] found a poor correlation between fluid-induced changes in Ea and those in ESP (ESP was estimated as $0.9 \times \mathrm{SAP}$ ), and concluded that Ea should

Table 4 Univariate logistic regression analysis for screening potential predictors of stroke volume response to norepinephrine

\begin{tabular}{|c|c|c|c|c|}
\hline \multirow[t]{2}{*}{ Variables } & \multirow{2}{*}{$\begin{array}{l}\text { Odd } \\
\text { ratio }\end{array}$} & \multicolumn{2}{|c|}{$95 \% \mathrm{Cl}$ for odd ratio } & \multirow{2}{*}{$\begin{array}{l}P \\
\text { value }\end{array}$} \\
\hline & & Lower & Upper & \\
\hline HR (beats/min) & 0.983 & 0.945 & 1.021 & 0.374 \\
\hline SAP (mmHg) & 0.934 & 0.832 & 1.048 & 0.244 \\
\hline $\mathrm{DAP}(\mathrm{mmHg})$ & 0.956 & 0.832 & 1.098 & 0.522 \\
\hline MAP (mmHg) & 0.906 & 0.763 & 1.077 & 0.264 \\
\hline CVP (mmHg) & 0.941 & 0.792 & 1.117 & 0.486 \\
\hline LVEDV (mL) & 0.966 & 0.911 & 1.025 & 0.257 \\
\hline LVEF (\%) & 1.154 & 1.028 & 1.296 & 0.015 \\
\hline $\mathrm{SV}(\mathrm{mL})$ & 1.042 & 0.976 & 1.114 & 0.218 \\
\hline $\mathrm{VTI}(\mathrm{cm})$ & 1.302 & 1.032 & 1.643 & 0.026 \\
\hline NE dose $(\mu \mathrm{g} / \mathrm{kg} / \mathrm{min})$ & 1.778 & 0.469 & 6.738 & 0.397 \\
\hline Time from NE infusion start to MAP stabilization (min) & 1.000 & 0.994 & 1.006 & 0.940 \\
\hline $\mathrm{Ea} /$ Ees ratio & 0.008 & 0.000 & 0.293 & 0.009 \\
\hline
\end{tabular}

SV stroke volume; NE norepinephrine; HR heart rate; SAP systolic arterial pressure; DAP diastolic arterial pressure; MAP mean arterial pressure; CVP central venous pressure; VTI velocity-time integral; LVEDV left ventricular end-diastolic volume; LVEF left ventricular ejection fraction; Ea effective arterial elastance; Ees left ventricular effective end-systolic elastance; $\mathrm{Cl}$ confidence interval 


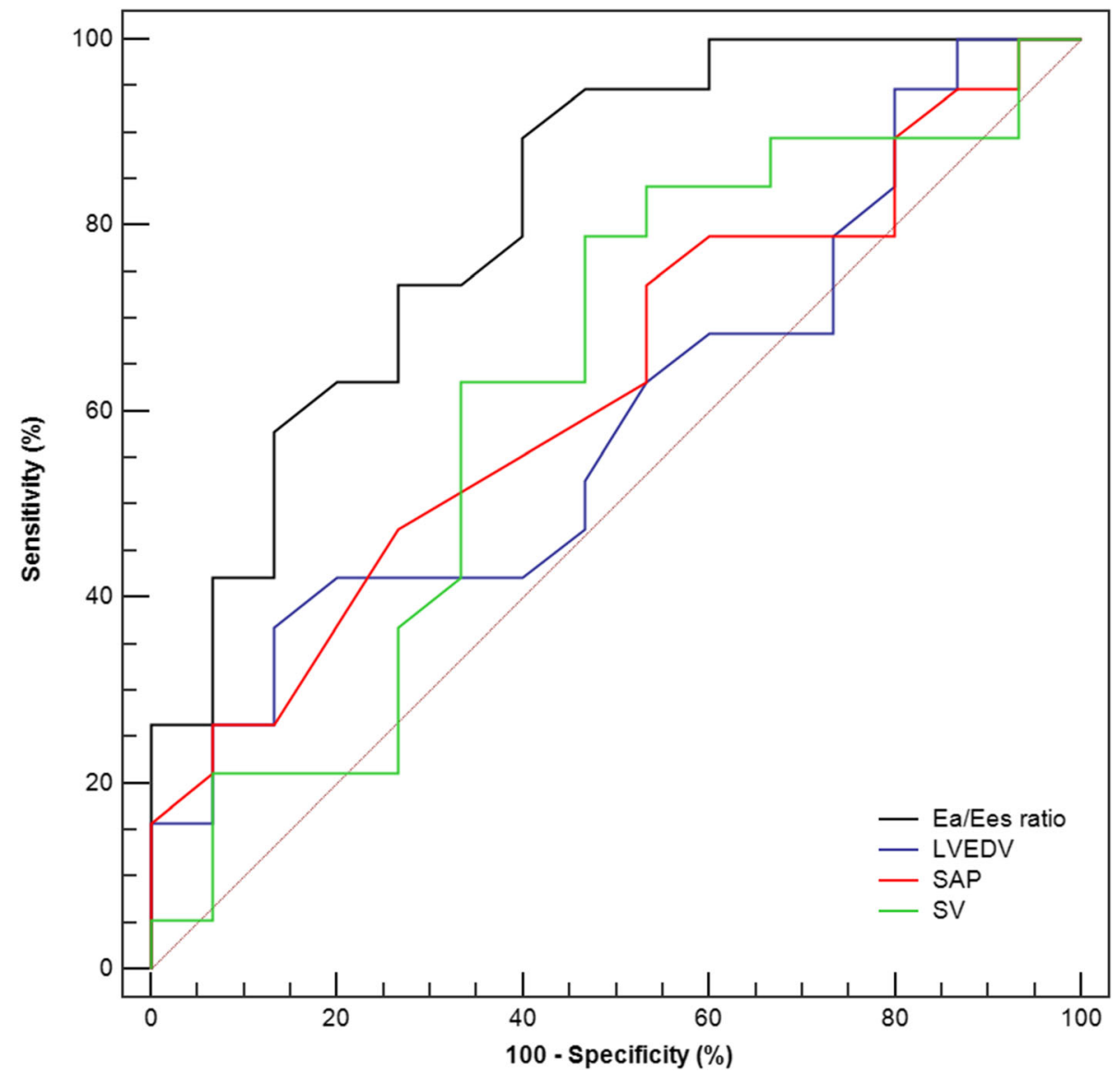

Fig. 4 Receiver operating characteristic curves to discriminate stroke volume response to norepinephrine. Ea effective arterial elastance; Ees left ventricular effective end-systolic elastance; SV stroke volume; SAP systolic arterial pressure; LVEDV left ventricular end-diastolic volume

not be used in isolation as an index of left ventricular afterload. Inconsistent with the previous study, the current one indicated that the NE-induced increases in Ea were related well to the NE-induced increases in SAP $(r=0.802, P<0.01)$. Different interventions might be a potential explanation for these conflicting findings. In their study, fluid loading primarily increased the SV and thus led to a reduction of $\mathrm{Ea}$, despite the increases in SAP. Conversely, in our study, NE increased the Ea by improving the SAP through its main vasoconstrictive effect. Based on these findings, whether Ea can be considered as an index of ventricular afterload still needs more discussion.

The current study has a main clinical implication that the evaluation of left VAC before NE infusion is helpful to identify which population will benefit from the use of NE. Maintenance of perfusion pressure while still sustaining adequate $\mathrm{CO}$ is crucial for hypotensive patients [9]. Theoretically, among hypotensive patients treated with NE to restore the arterial pressure, those patients with increased SV after NE infusion may suffer from better clinical prognosis than those with unchanged or decreased SV. Our study indicates that septic shock patients with a baseline left VAC $>1.11$ are more likely to improve the SV with use of NE. For those septic shock patients with a baseline left $\mathrm{VAC}<1.11$, the abuse of a large dose of $\mathrm{NE}$ should be avoided because its great afterload effects on the left ventricle might reduce the SV. Accordingly, our study provides a new perspective that dynamic assessment of left VAC during the resuscitation of septic shock may be a promising monitoring strategy to guide titrated adjustment of NE dosage to optimize the cardiac work efficacy and thus improve clinical prognosis.

There are several limitations to our study. Firstly, the small fluid volume administered during the study period might affect the left VAC to a small extent. While we had restricted changes in some variables that might affect the left VAC (e.g. IMV setting, fluid challenge), the fluid administration was not completely restricted during the study period because it was unrealistic in the clinical practice due to the relatively long study period (median duration of $95 \mathrm{~min}$ ). Secondly, IMV and sedative and analgesic drugs may also be confounders 
affecting the left VAC due to its hemodynamic effects [28-30]. Unfortunately, we did not analyze the IMV parameters and the dose of sedatives and analgesics because of the limited sample size. Nevertheless, the use of IMV and sedatives or analgesics would not prevent the deduction of the conclusion, because modifications of these variables were not allowed during the study period.

Lastly, as discussed previously $[19,23,25]$, the method used for the estimation of Ea and Ees remains a challenge for the reliability of our findings. Estimation of Ees in our study was based on the noninvasive single-beat method [21] that assumed a load-independent linear end-systolic pressure-volume relationship and a constant volume axis intercept (V0) of the relationship curve. However, a previous study reported a significant correlation between the V0 and cardiac function [14]. Thus, changes in V0 resulted from impaired cardiac contractility might affect the estimation of Ees. Furthermore, we measured the radial arterial pressure as a surrogate of aortic systolic pressure to calculate the Ea. However, the radial arterial pressure was reported to be less accurate than the femoral arterial pressure to estimate the Ea [26] and it may be imprecise to represent the aortic systolic pressure in septic shock due to the collapsed circulatory system [23]. Even so, it would not affect the precision of calculation of Ea/Ees ratio because of the similar influences on the calculation of Ea and Ees. Thus, the left $\mathrm{VAC}$ can be considered as a valid predictor of SV response to NE.

\section{Conclusions}

Administration of NE induced changes in Ea and Ees in patients with septic shock. The SV response to NE was determined by the comprehensive effects of norepinephrine on the left VAC, which depended on the left VAC at baseline. The baseline left VAC had predictive value for the $\mathrm{SV}$ response to NE infusion in patients with septic shock.

\section{Abbreviations}

ICU: intensive care unit; NE: norepinephrine; CO: cardiac output; DAP: diastolic arterial pressure; SV: stroke volume; VAC: ventricular-arterial coupling; Ea: effective arterial elastance; Ees: left ventricular effective endsystolic elastance; CVP: central venous pressure; LVEF: left ventricular ejection fraction; MAP: mean arterial pressure; TTE: transthoracic echocardiography; IMV: invasive mechanical ventilation; APACHE: acute physiology and chronic health evaluation; SOFA: sequential organ failure assessment; $\mathrm{PaO} 2$ : arterial oxygen partial pressure; FiO2: fractional inspired oxygen; LVEDV: left ventricular end-diastolic volume; LVESV: left ventricular end-systolic volume; $V T$ : aortic velocity-time integral; $T_{\text {pre-e: }}$ pre-ejection time; $T_{\text {tot-s: }}$ total systolic time; LVOT: left ventricular outflow tract; HR: heart rate; SAP: systolic arterial pressure; ESP: end systolic pressure; SD: standard deviation; IQR: interquartile range; ROC: receiver operating characteristic; AUC: area under the ROC curve; $\mathrm{CV}$ : coefficient of variation

\section{Authors' contributions}

XZ designed the study, enrolled patients, analyzed and interpreted data, and drafted the manuscript. JP enrolled patients, performed the statistical analysis, and helped to acquire and interpret data. YW and HW enrolled patients, acquired data, and helped to perform the statistical analysis. ZX and WZ designed the study, analyzed and interpreted data, and revised the manuscript. All authors read and approved the final manuscript.

\section{Funding}

This study was supported by the grants from Zhejiang Medicine and Health Science and Technology Project (No. 2019KY184) and Natural Science Foundation of Zhejiang Province (No. LY19H190001). The funders had no role in the design of the study or collection, analysis, or interpretation of data or writing the manuscript.

\section{Availability of data and materials}

The datasets used and/or analyzed during the current study are available from the corresponding author on reasonable request.

\section{Ethics approval and consent to participate}

This study was approved by the institutional ethics committee in HwaMei Hospital, University of Chinese Academy of Sciences. Written informed consent was obtained from the patients or their next of kin.

\section{Consent for publication}

Not applicable.

\section{Competing interests}

The authors declare that they have no competing interests.

\section{Author details}

${ }^{1}$ Department of Intensive Care Medicine, HwaMei Hospital, University of Chinese Academy of Sciences, Ningbo, Zhejiang 315000, China. ${ }^{2}$ Ningbo Institute of Life and Health Industry, University of Chinese Academy of Sciences, Ningbo, Zhejiang 315000, China. ${ }^{3}$ Department of Intensive Care Medicine, Ningbo Fenghua District Hospital of Traditional Chinese Medicine Medical Community, Ningbo, Zhejiang 315500, China.

Received: 19 September 2020 Accepted: 28 January 2021

Published online: 17 February 2021

\section{References}

1. Vincent $J L$, Jones $G$, David S, Olariu E, Cadwell KK. Frequency and mortality of septic shock in Europe and North America: a systematic review and meta-analysis. Crit Care. 2019;23(1):196.

2. Hamzaoui O, Shi R. Early norepinephrine use in septic shock. J Thorac Dis. 2020;12(Suppl 1):S72-7.

3. Scheeren TWL, Bakker J, De Backer D, Annane D, Asfar P, Boerma EC, et al. Current use of vasopressors in septic shock. Ann Intensive Care. 2019;9(1):20.

4. Levy MM, Evans LE, Rhodes A. The surviving Sepsis campaign bundle: 2018 update. Intensive Care Med. 2018;44(6):925-8.

5. Rhodes A, Evans LE, Alhazzani W, Levy MM, Antonelli M, Ferrer R, et al. Surviving Sepsis campaign: international guidelines for Management of Sepsis and Septic Shock: 2016. Intensive Care Med. 2017:43(3):304-77.

6. Hernández G, Teboul JL, Bakker J. Norepinephrine in septic shock. Intensive Care Med. 2019;45(5):687-9.

7. Espinoza EDV, Hernandez G, Bakker J. Norepinephrine, more than a vasopressor. Ann Transl Med. 2019; 7(Suppl 1): S25.

8. Persichini R, Silva S, Teboul JL, Jozwiak M, Chemla D, Richard C, et al. Effects of norepinephrine on mean systemic pressure and venous return in human septic shock. Crit Care Med. 2012;40(12):3146-53.

9. Maas JJ, Pinsky MR, de Wilde RB, de Jonge E, Jansen JR. Cardiac output response to norepinephrine in postoperative cardiac surgery patients: interpretation with venous return and cardiac function curves. Crit Care Med. 2013;41(1):143-50

10. Guarracino F, Baldassarri R, Pinsky MR. Ventriculo-arterial decoupling in acutely altered hemodynamic states. Crit Care. 2013:17(2):213.

11. Binkley PF, Van Fossen DB, Nunziala E, Unverferth DV, Leier CV. Influence of positive inotropic therapy on pulsatile hydraulic load and ventricularvascular coupling in congestive heart failure. J Am Coll Cardiol. 1990;15(5):1127-35. 
12. Elzinga G, Westerhof N. Matching between ventricle and arterial load. Circ Res. 1991;68(6):1495-500

13. Yan J, Zhou X, Hu B, Gong S, Yu Y, Cai G, et al. Prognostic value of left ventricular-arterial coupling in elderly patients with septic shock. J Crit Care. 2017:42:289-93.

14. Ky B, French B, May Khan A, Plappert T, Wang A, Chirinos JA, et al. Ventricular-arterial coupling, remodeling, and prognosis in chronic heart failure. J Am Coll Cardiol. 2013;62(13):1165-72.

15. Antonini-Canterin F, Enache R, Popescu BA, Popescu AC, Ginghina C, Leiballi $E$, et al. Prognostic value of ventricular-arterial coupling and B-type natriuretic peptide in patients after myocardial infarction: a five-year followup study. J Am Soc Echocardiogr. 2009;22(11):1239-45.

16. Huang SJ. Measuring cardiac output at the bedside. Curr Opin Crit Care. 2019;25(3):266-72.

17. Ikonomidis I, Aboyans V, Blacher J, Brodmann M, Brutsaert DL, Chirinos JA, et al. The role of ventricular-arterial coupling in cardiac disease and heart failure: assessment, clinical implications and therapeutic interventions. A consensus document of the European Society of Cardiology Working Group on Aorta \& Peripheral Vascular Diseases, European Association of Cardiovascular Imaging, and heart failure association. Eur J Heart Fail. 2019; 21(4):402-24.

18. Singer M, Deutschman CS, Seymour CW, Shankar-Hari M, Annane D, Bauer $M$, et al. The third international consensus definitions for Sepsis and septic shock (Sepsis-3). JAMA. 2016;315(8):801-10.

19. Guinot $P G$, Longrois $D$, Kamel S, Lorne E, Dupont H. Ventriculo-arterial coupling analysis predicts the hemodynamic response to norepinephrine in hypotensive postoperative patients: a prospective observational study. Crit Care Med. 2018;46(1):e17-25.

20. Kelly RP, Ting CT, Yang TM, Liu CP, Maughan WL, Chang MS, et al. Effective arterial elastance as index of arterial vascular load in humans. Circulation. 1992;86(2):513-21.

21. Chen CH, Fetics B, Nevo E, Rochitte CE, Chiou KR, Ding PA, et al. Noninvasive singlebeat determination of left ventricular end-systolic elastance in humans. J Am Coll Cardiol. 2001;38(7):2028-34.

22. Chen CH, Nakayama M, Nevo E, Fetics BJ, Maughan WL, Kass DA. Coupled systolic-ventricular and vascular stiffening with age: implications for pressure regulation and cardiac reserve in the elderly. J Am Coll Cardiol. 1998;32(5): $1221-7$.

23. Guarracino F, Ferro B, Morelli A, Bertini P, Baldassarri R, Pinsky MR. Ventriculoarterial decoupling in human septic shock. Crit Care. 2014;18(2):R80.

24. Hamzaoui O, Jozwiak M, Geffriaud T, Sztrymf B, Prat D, Jacobs F, et al. Norepinephrine exerts an inotropic effect during the early phase of human septic shock. Br J Anaesth. 2018;120(3):517-24.

25. Huette $\mathrm{P}, \mathrm{Abou}$-Arab $\mathrm{O}$, Longrois $\mathrm{D}$, Guinot $\mathrm{PG}$. Fluid expansion improve ventriculo-arterial coupling in preload-dependent patients: a prospective observational study. BMC Anesthesiol. 2020;20(1):171.

26. Jozwiak M, Millasseau S, Richard C, Monnet X, Mercado P, Dépret F, et al. Validation and critical evaluation of the effective arterial Elastance in critically ill patients. Crit Care Med. 2019;47(4):e317-24.

27. Monge García Ml, Santos A. Understanding ventriculo-arterial coupling. Ann Transl Med. 2020;8(12):795.

28. Dres M, Teboul JL, Monnet $X$. Weaning the cardiac patient from mechanical ventilation. Curr Opin Crit Care. 2014;20(5):493-8.

29. Deryck YL, Fonck K, DE Baerdemaeker L, Naeije R, Brimioulle S. Differential effects of sevoflurane and propofol anesthesia on left ventricular-arterial coupling in dogs. Acta Anaesthesiol Scand. 2010;54(8):979-86.

30. Pittarello D, Bonato R, Marcassa A, Pasini L, Falasco G, Giron GP. Ventriculoarterial coupling and mechanical efficiency with remifentanil in patients with coronary artery disease. Acta Anaesthesiol Scand. 2004;48(1): $61-8$

\section{Publisher's Note}

Springer Nature remains neutral with regard to jurisdictional claims in published maps and institutional affiliations.

Ready to submit your research? Choose BMC and benefit from:

- fast, convenient online submission

- thorough peer review by experienced researchers in your field

- rapid publication on acceptance

- support for research data, including large and complex data types

- gold Open Access which fosters wider collaboration and increased citations

- maximum visibility for your research: over $100 \mathrm{M}$ website views per year

At $\mathrm{BMC}$, research is always in progress.

Learn more biomedcentral.com/submissions 